\title{
Studies of Low-Frequency Power Amplifier Class D with Operational Amplifiers and Transistors
}

\author{
Karapenev Boyan Dimitrov \\ Department of the Communication Equipment and Technologies, Technical University of Gabrovo, Gabrovo, Bulgaria \\ Email address: \\ bkarapenev@tugab.bg \\ To cite this article: \\ Karapenev Boyan Dimitrov. Studies of Low-Frequency Power Amplifier Class D with Operational Amplifiers and Transistors. Journal of \\ Electrical and Electronic Engineering. Vol. 5, No. 5, 2017, pp. 170-179. doi: 10.11648/j.jeee.20170505.13
}

Received: September 14, 2017; Accepted: September 25, 2017; Published: October 13, 2017

\begin{abstract}
This paper presents studies of Low-Frequency Power Amplifier class D with operational amplifiers and transistors. Their features have been presented and a selected circuit is described. The obtained results have been presented using MultiSIM software - oscillograms in specific nodes of the circuit, amplitude-frequency and phase-frequency responces and their parameters, and results of carried out Fourier and Parameter Sweep analyzes. Simulation results could be used in carrying out the optimization of its characteristics and qualitative parameters, as well as present and expected practical results. The results of performed experimental studies of implemented low-frequency power amplifier class D are presented also.
\end{abstract}

Keywords: Low-Frequency Power Amplifier Class D, Studies, Qualitative Parameters

\section{Introduction}

The development of electronics is inextricably linked to the use of amplification equipment in all its frequency and power ranges. This necessitates an increase of the useful output power and an increase of the efficiency. Lowfrequency power amplifiers class $\mathrm{D}$ have low dissipation power and heat dissipation, small size and price, minimum power consumption in idle mode. The main advantage of class D amplifiers is the high efficiency, which can reach 90 $\div 98 \%[7]$.

Low-frequency power amplifiers class D have been in existence for more than a few decades and are currently used mainly at low frequencies and high powers, i.e. for subwoofer management, and more rarely for amplifying middle and high frequencies of the sound range due to the significant distortions associated with the imperfections of the switching technology used. They are also used as low frequency amplifiers in modern television receivers, in audio- and home cinema systems, GSM communications, and more [10].

\section{Representation}

Class D amplifiers operate in switch mode with two states of the active element: unstopped, when a maximum current flows through the negligible low impedance of the saturated final transistor for a very short time interval with minimum losses, and clogged - when the current is approximately zero and the voltage drop is almost equal to the supply voltage without losses on the transistor. The main losses on the active element are mainly due to the time of impulse formation, i.e. when switching from one to the other state. If the duration of the fronts is reduced to zero, the efficiency will approach up to $100 \%$ [1]. This is the case when the active electronic element is replaced by an ideal switch. Such an idealized mode is not achievable due to the presence of: a minimum voltage on the saturated transistors $U_{\text {CEsat }}$ in the fully unstopped state, the reverse current in clogged state and the impossibility for the switching time to be infinitely small [6]. This time is in the range of one (or less) to tens of microseconds. To make possible the usage of the most efficient mode of operating the transistors, the switch, it is necessary to use a certain type of modulation. Of the possible modes for modulation in this mode of operation of the devices, the pulse width modulation (PWM) [3] is the most convenient in which the pulse duration varies according to the amplitude of the input modulation signal. 


\subsection{A Circuit of Low-Frequency Power Amplifier Class D with Operational Amplifiers and Transistors}

A circuit of Low-Frequency Power Amplifier (LFPA) class $\mathrm{D}$ with operational amplifiers (OA) and transistors is presented in Figure 1. It contains four preliminary stages with operational amplifiers, two driver stages built with push-pull connected bipolar transistors, final MOSFET transistors designed for class $\mathrm{D}$ operation and a simplified configuration of the ouput low-pass (LC) filter used. The circuit is suitable for simulation study and practical implementation, and the results obtained illustrate its principle of operation.

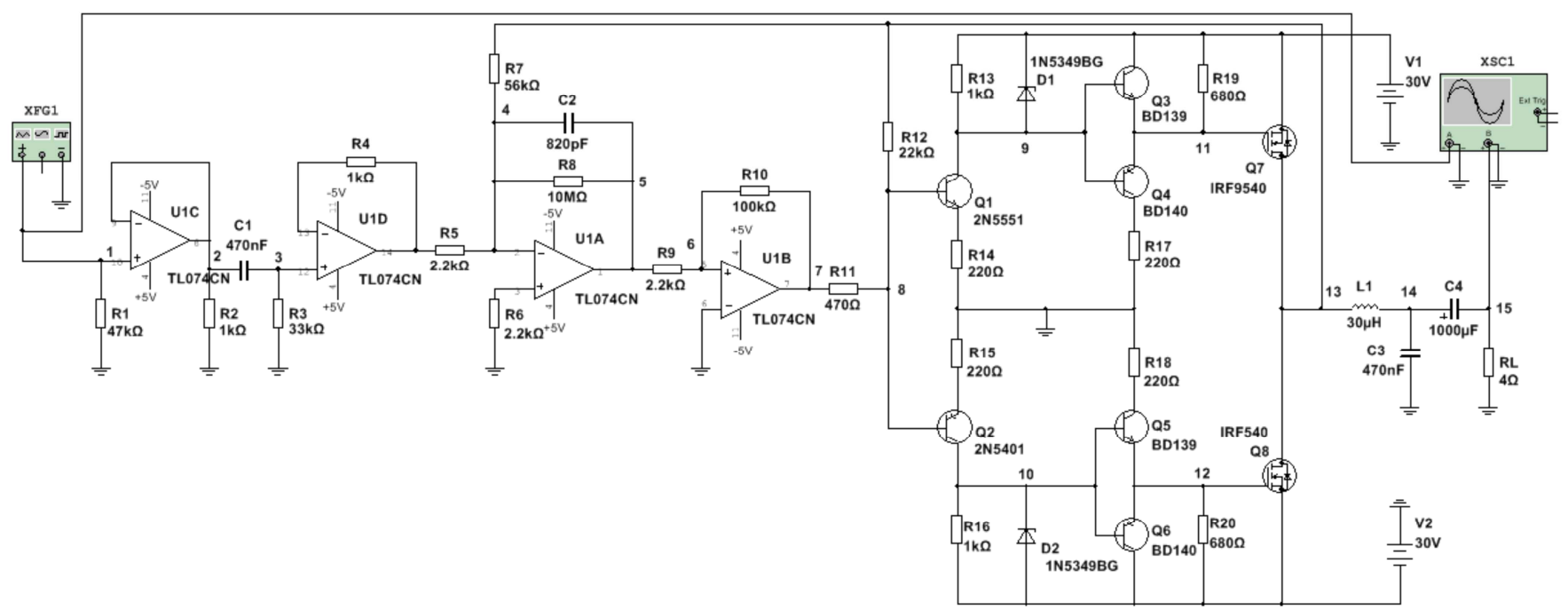

Figure 1. A Circuit of Low-frequency Power Amplifier Class D with Operational Amplifiers and Transistors.

The input stage is implemented with a non-inverting voltage repeater with $\mathrm{OA} 1$ (U1C) as the gain ratio is $\mathrm{A}_{\mathrm{UF}} \approx 1$. The second OA2 (U1D) is also connected as a non-inverting amplifier with $\mathrm{A}_{\mathrm{UF}}=1+\mathrm{R} 4 / \mathrm{R} 3$, and the capacitor $\mathrm{C} 1$ serves as blocking between the two input stages. These two input stages perform the function of buffer amplifiers.

OA3 (U1A) has a complex function by performing a "summing" of its inverting input (adder-integrator) of the input signal with the output PWM signals formed at the output of the final stage (node 13) via the inverse feedback $\mathrm{R} 7$ as a result of which the output voltage is triangular in shape. Its voltage gain is determined by the ratio of the values of $\mathrm{R} 8$ and $\mathrm{R} 5$, and the capacitor $\mathrm{C} 2$ also provides a frequency correction for the OA. OA4 (U1B) is a noninverting voltage comparator with positive feedback (saturated amplifier) realized with resistor R10 connected between its output and non-inverting input. The two stages, comprising OA3 and OA4, realize the implementation of the pulse width modulation and together they form a PWMcontroller.

The final MOSFET transistors Q7 and Q8, coupled by OD schemes, of the $\mathrm{P}$ and $\mathrm{N}$ type respectively, form a semiconductor circuit.

The output low-pass filter is realized with the L1 - C3 elements.

The output amplified modulated voltage with PWM is applied to the inverting input of OA3 and the base of the transistors Q1 and Q2 respectively via the resistors R7 and R12 performing the functions of interstage parallel inverse feedbacks by voltage.

The formed width-modulated pulses at the output OA4 (U1B) unstop sequentially the transistors Q1-Q2, Q3-Q4 -
Q5-Q6 in the presence of an input (modulating) signal and because the potentials $\mathrm{U}_{\mathrm{GS}}$ of the final MOSFETs Q7 and Q8 are increasing, the latter are also unstopped.

\subsection{Simulation Studies of Low-Frequency Power Amplifier Class D with Operational Amplifiers and Transistors}

The principle diagram (circuit) of the LFPA class D with the operational amplifiers and transistors from Figure 1 is introduced into the MultiSIM module of the software Circuit Design Suite for which simulation studies have been performed. The set parameters of the input sinusoidal signal through the functional generator used are: frequency $1 \mathrm{kHz}$, amplitude $1 \mathrm{~V}_{\mathrm{p}}$ and displacement of the DC current level - 0 .

Figure 2 illustrates how OA3 performs a "summing" of its inverting input (adder-integrator) of the input signal with the output PWM signals formed at the output of the final stage (node 13) via the inverse feedback R7, as a result of which the output voltage is triangular in shape and follows the change of the input continuous signal.

In Figure 3 is presented the oscillogram of the signal entering the transistor bases Q1 and Q2 which have the function of forming the amplitude of the PWM highfrequency signal. The signal is slightly increased compared to that of node 7 since the R12 returns a part of the output amplified PWM signal. The pulses of the OA4 output have a duty factor of about $50 \%$ with a constant amplitude in the field of passing of the input signal through the zero level. At the maximum value of the input signal, there is a minimum pulse width and amplitude reduction, and at the minimum - the inverse. A rebound of the front and rear impulse faces is seen on the oscillogram of the output signal in Figure 3. 
In Figure 4, the signal was already been amplified and enters the base of the driver transistors Q3 and Q4. The PWM signal is displaced at a constant current level (node 9), which has a displacement $\mathrm{Y}$ pos. (div): $-4,8$ on the oscillogram in the "upper" arm of the circuit. The rectangular pulses have the same amplitude for the negative side of the input sinusoidal signal unlike the oscillograms in node 7 and Figure 3.

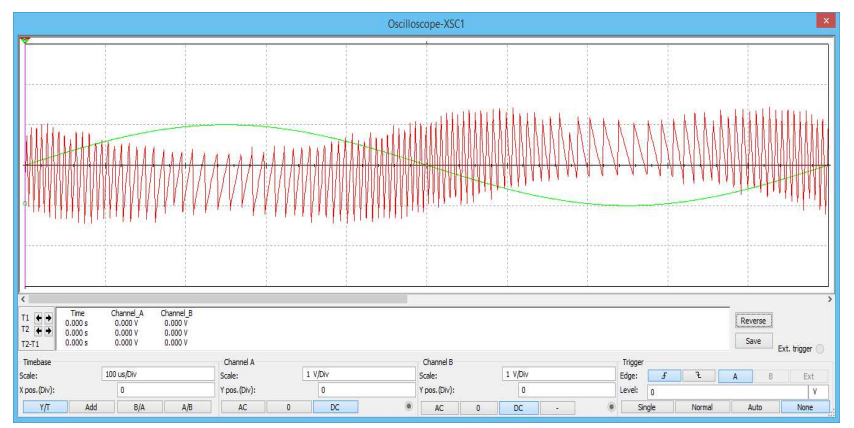

Figure 2. Input Signal (in Green) and Output Signal of OA3 (in Red) - Node 5 .

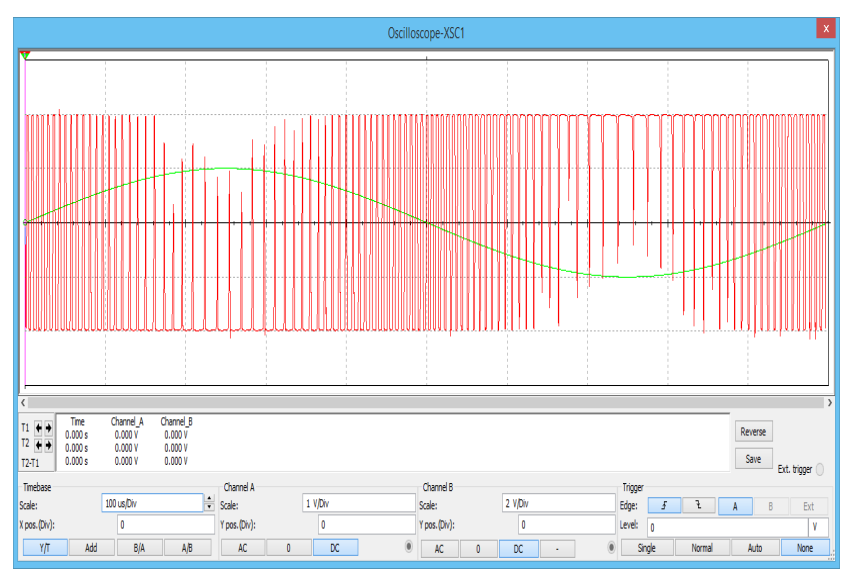

Figure 3. Input Signal (in Green) and Base Signal of Transistors $Q 1$ and Q2 (in Red) - Node 8.

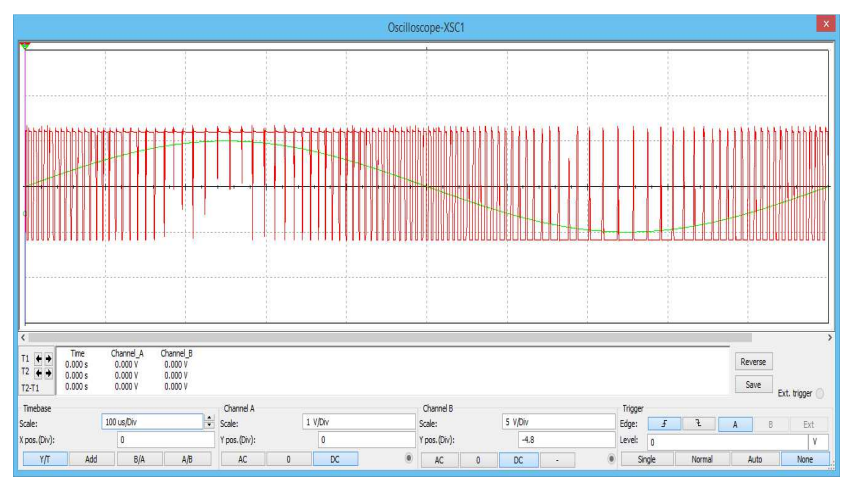

Figure 4. Input Signal (in Green) and Base Signal of Transistors $Q 3$ and $Q 4$ (in Red) - Node 9.

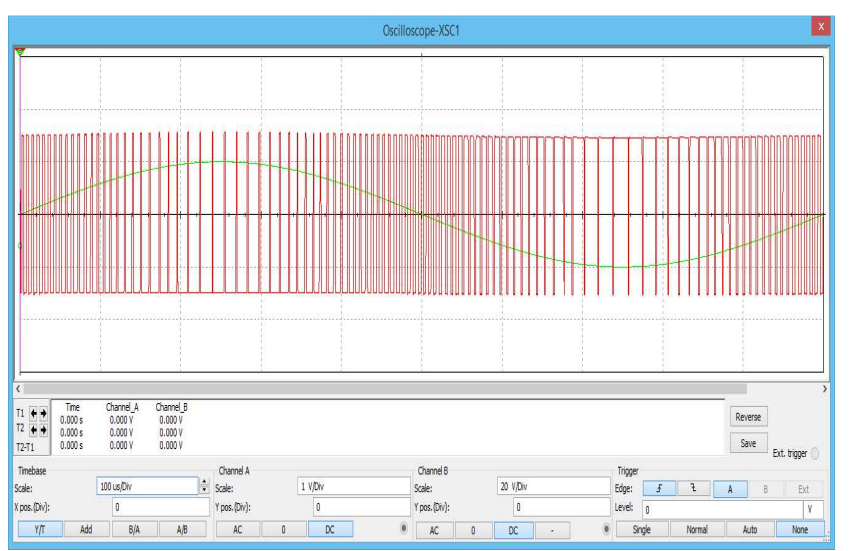

Figure 5. Input Signal (in Green) and Draine Signal of Transistors $Q 7$ and Q8 (in Red) - Node 13.

In Figure 5 is given the oscillogram of amplified signal of the drains of the final MOSFET transistors Q7 and Q8. This is a PWM signal that combines the impulses of the final MOSFET transistors, where the signals are with constant amplitude and compensated by the two arms distortions of overshoots of the front and rear of the impulse faces.

The output signal of the LFPA with operational amplifiers and transistors is with low frequency and contains high frequency comprising components, resembling a high frequency carrier oscillation and a signal shape close to the sinusoidal one and is phased at $180^{\circ}$ to the input continuous signal.

From the carred out DC analysis (DC Operation Point) of the LFPA class D has been established that the DC current potentials in the individual nodes have values in the range of tens of $\mu \mathrm{V}$ and since the OA4 and the transistors $\mathrm{Q} 1 \div \mathrm{Q} 8$ are in swich mode, there are no potentials for establishing the circuit in DC mode.

Figure 6 and Figure 7 present the obtained results of the performed Alternating Current analysis (AC Frequency) for the LFPA - respectively amplitude- and phase- frequency responses.

From Figure 6 the parameters of the LFPA are set: lower $f_{\mathrm{b}}$ $=10 \mathrm{~Hz}$, high $f_{\mathrm{h}}=28,75 \mathrm{kHz}$ cut-off frequencies and transmitted frequency band (bandwidth) $\Delta f=f_{\mathrm{h}}-f_{\mathrm{b}}=28,74$ $\mathrm{kHz}$.

On the shown phase-frequency response (Figure 7) is seen a transition with a phase shift of $-180^{\circ}$ to $+180^{\circ}$, i.e. at $360^{\circ}$ of the output signal for frequencies between 389 and $507 \mathrm{~Hz}$. This could satisfy the condition of phase angle balance and the LFPA class D circuit to become a oscillator.

The results of the obtained Fourier Analysis are presented in Figure 8 where in tabular form are presented the DC component, number of harmonics, full harmonic distortion factor (THD) as well as the real and imaginary part of the individual harmonics and their normalized values.

With the thus obtained values of the amplitudes of the individual harmonic components from the spectrum of the output signal of the LFPA class D (Figure 8, Magnitude) the coefficient of non-linear distortions is determined for which the obtained value is 

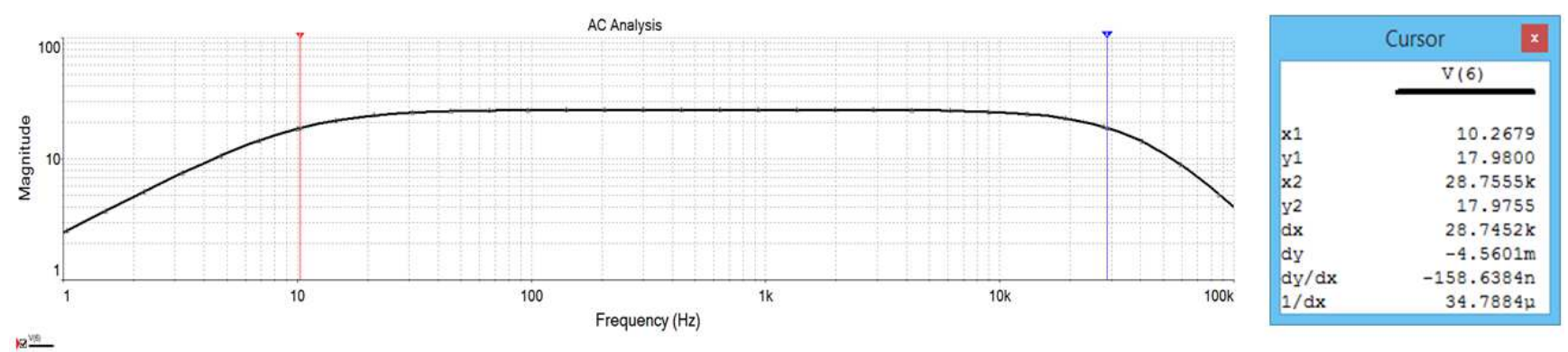

Figure 6. Amplitude-Frequency Response of LFPA Class D with Operational Amplifiers and Transistors.
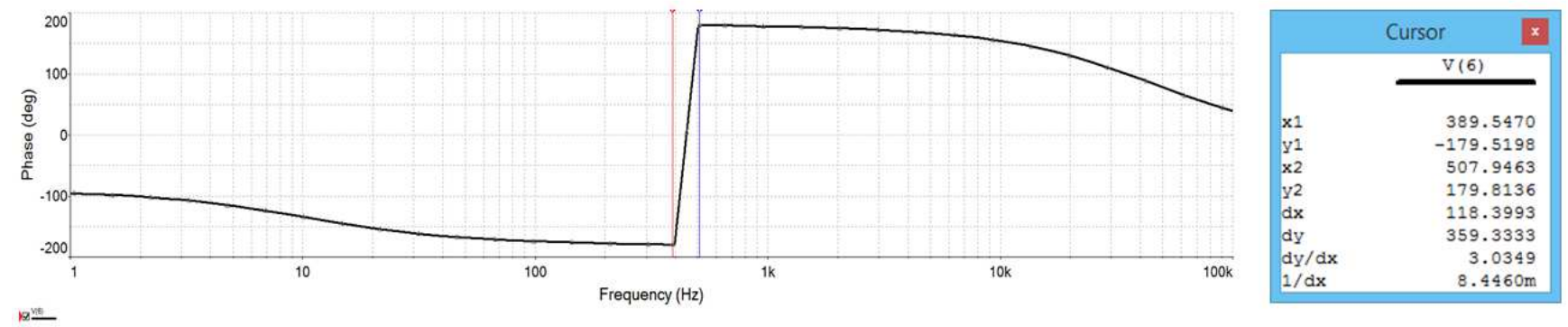

Figure 7. Phase-Frequency Response of LFPA Class D with Operational Amplifiers and Transistors.

Fourier Analysis

\begin{tabular}{|c|c|c|c|c|c|c|}
\hline & & & & & & \\
\hline \begin{tabular}{l|l}
1 & $F$ \\
\end{tabular} & Fourier analysis for $\mathrm{V}(6)$ : & & & & & \\
\hline $2 D$ & DC component: & 0.249727 & & & & \\
\hline $3 \mathrm{~N}$ & No. Harmonics: & 9 & & & & \\
\hline 4 T & THD: & $0.615826 \%$ & & & & \\
\hline $5 G$ & Grid size: & 256 & & & & \\
\hline 6 Ir & Interpolation Degree: & 1 & & & & \\
\hline 7 & & & & & & \\
\hline $8 \mathrm{H}$ & Harmonic & Frequency & Magnitude & Phase & Norm. Mag & Norm. Phase \\
\hline $\begin{array}{lll}9 & 1\end{array}$ & & 1000 & 25.4254 & 177.54 & 1 & 0 \\
\hline 102 & & 2000 & 0.0194284 & 159.788 & 0.000764135 & -17.752 \\
\hline 113 & & 3000 & 0.0898278 & 56.5093 & 0.003533 & -121.03 \\
\hline 12 & & 4000 & 0.0377477 & 175.691 & 0.00148465 & -1.8484 \\
\hline 135 & & 5000 & 0.0549667 & -76.054 & 0.00216188 & -253.59 \\
\hline 146 & & 6000 & 0.0402929 & -62.62 & 0.00158475 & -240.16 \\
\hline 157 & & 7000 & 0.0711598 & -174.15 & 0.00279877 & -351.69 \\
\hline 168 & & 8000 & 0.066207 & -70.83 & 0.00260397 & -248.37 \\
\hline 179 & & 9000 & 0.0235104 & -10.12 & 0.000924682 & -187.66 \\
\hline
\end{tabular}

Figure 8. Results of the Fourier Analysis Obtained.

$$
\begin{gathered}
k=\frac{\sqrt{U_{2}^{2}+U_{3}^{2}+U_{4}^{2}+U_{5}^{2}+U_{6}^{2}+U_{7}^{2}+U_{8}^{2}+U_{9}^{2}}}{U_{1}}= \\
=\frac{\sqrt{0,02419}}{25,4}=0,006123 \cdot 100=0,6123 \% .
\end{gathered}
$$

The obtained value of $k=0,6123 \%$ is small since the amplitude of the first harmonic component is significantly greater than that of the others, which determines that the LFPA class D in Figure 1 is high quality - Hi-Fi.

The relative difference between the $k$ value and the THD parameter (Figure 8 ) is

$$
\varepsilon=\frac{k-T H D}{T H D}=\frac{0,6123-0,6158}{0,6158}="-" 0,00568 \% .
$$

Using the Parameter Sweep Analysis in Figure $9 \div$ Figure 11 is shown the influence of the values of the elements R7, R12 (from inverse feedback) and C2 (introducing lowfrequency correction of OA3) on the Amplitude-frequency response of the LFPA class D with operational amplifiers and transistors, respectively.

It is seen from Figure 9, that the value of the R7 resistor of the inverse feedback changes the gain ratio (the amplitude of the output signal), the slope of the decrease of the Amplitude-frequency response and the values of the $f_{\mathrm{b}}, f_{\mathrm{h}}$ and $\Delta f$. The obtained parameters of the LFPA class D from Figure 1 for some of the set values of the R7 resistor are presented in Table 1. 


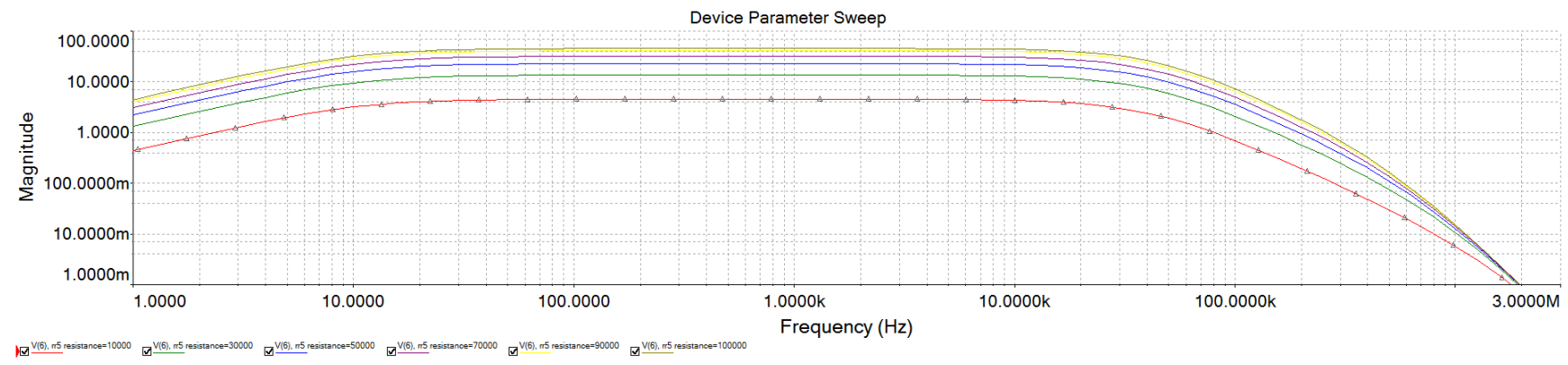

Figure 9. Parameter Sweep Analysis Relative to the R7 Value.

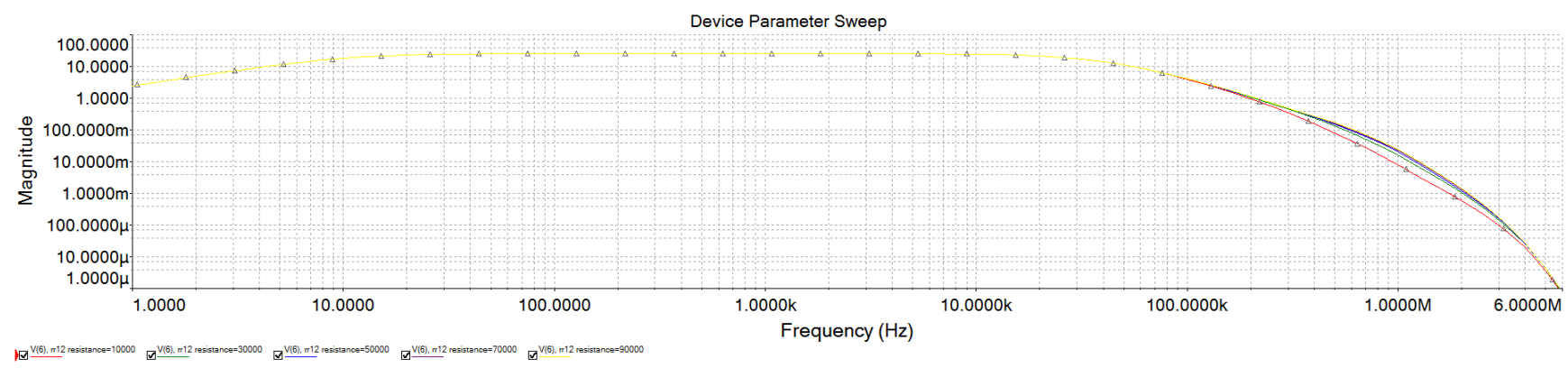

Figure 10. Parameter Sweep Analysis Relative to the R12 Value.

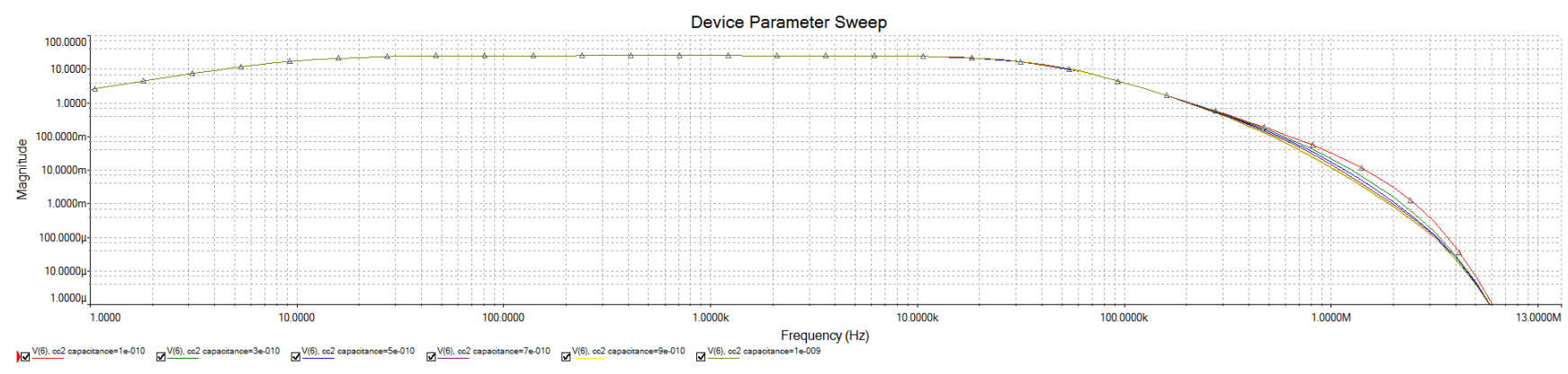

Figure 11. Parameter Sweep Analysis Relative to the C2 Value.

Table 1. Qalitative Parameters of the LFPA Class D at Different Values of the $R 7$ Resistor of the Inverse Feedback.

\begin{tabular}{llllll}
\hline Value of $\mathbf{R} 7, \mathbf{k} \boldsymbol{\Omega}$ & $\mathbf{U}_{\mathbf{0}}, \mathbf{V}$ & $\mathbf{A}_{\mathbf{U}}$ & $\boldsymbol{f}_{\mathbf{b}}, \mathbf{H z}$ & $\boldsymbol{f}_{\mathbf{h}}, \mathbf{k H z}$ & $\boldsymbol{\Delta} \boldsymbol{f}, \mathbf{k H z}$ \\
\hline 10 & 4,54 & 12 & 10,3 & 27,3 & 27,3 \\
30 & 13,6 & 38 & 10 & 28 & 28 \\
50 & 22,7 & 64 & 10 & 28,5 & 28,5 \\
100 & 45,4 & 128 & 10 & 32 & 32 \\
\hline
\end{tabular}

After graphically constructing the dependencies $\mathrm{A}_{\mathrm{U}}, f_{\mathrm{b}}, f_{\mathrm{h}}$ and $\Delta f$ in function of the value of the $\mathrm{R} 7$ resistor of the inverse feedback of LFPA class D has been found that:

a) the dependence of the gain ratio $A_{U}$ on the value of $R 7$ is linear;

b) smaller R7 values change insignificantly the value of $f_{b}$, and larger ones do not affect;

c) the dependencies of $\mathrm{f}_{\mathrm{h}}$ and $\Delta f$ are almost linear are coincide, since $f_{\mathrm{h}}$ determines the transmitted bandwidth.

The change in the values of the resistor R12 and the capacitor C2 have an impact on the Amplitude-frequency response of the LFPA class D only in the high frequency range, for frequencies above $200 \mathrm{kHz}$, outside the transmitted bandwidth. It is established that their influence is "opposite" i.e. the increase of the R12 value expands the fall of the Amplitude-frequency response in high frequency range and the increase of the $\mathrm{C} 2$ value narrows it.

\subsection{Experimental Studies of the Practical Implemented Low-frequency Power Amplifier Class D with Operational Amplifiers and Transistors}

Table 2 shows the DC voltages other than $0 \mathrm{~V}$ measured at the specified control nodes of the LFPA class D circuits at a supply voltage of $\pm \mathrm{U}_{\mathrm{CC}} 30 \mathrm{~V}$ and no input signal.

Table 2. DC Voltages in Respective Nodes of the LFPA Class D.

\begin{tabular}{llllllllll}
\hline Node № & $\mathbf{5}$ & $\mathbf{6}$ & $\mathbf{7}$ & $\mathbf{8}$ & $\mathbf{9}$ & $\mathbf{1 0}$ & $\mathbf{1 1}$ & $\mathbf{1 2}$ & $\mathbf{1 5}$ \\
\hline $\mathrm{U}, \mathrm{V}$ & $-0,1$ & $-0,1$ & 0,74 & 0,72 & 25,6 & $-25,8$ & 25,8 & -26 & $-0,07$ \\
\hline
\end{tabular}


The obtained amplitude (AP) and amplitude-frequency (AFR) characteristics of the LFPA class D are presented in Figure 12 and Figure 13 respectively, and the parameters determined by them - in Table 3 and Table 4, respectively.

Figure 12 shows that the implemented LFPA class D amplifies in the same way at the three operating frequencies from the performed experimental studies $-f_{1}=0,1 \mathrm{kHz}, f_{2}=$ $0,5 \mathrm{kHz}$ and $f_{3}=1 \mathrm{kHz}$, but the most linear is the dependence at medium frequencies $(1 \mathrm{kHz})$ where the dynamic range $\mathrm{D}$ takes the smallest value.

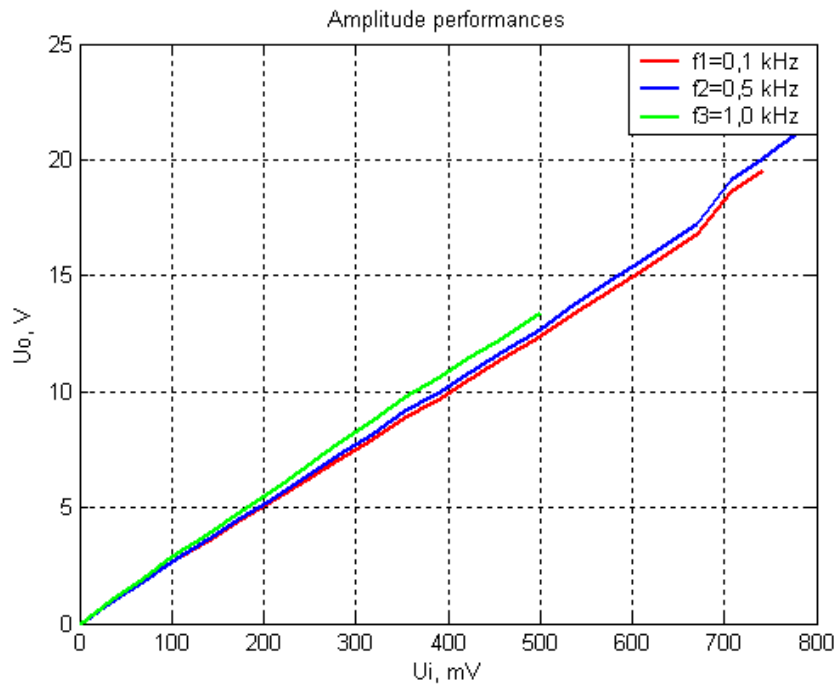

Figure 12. AP of the LFPA Class D.

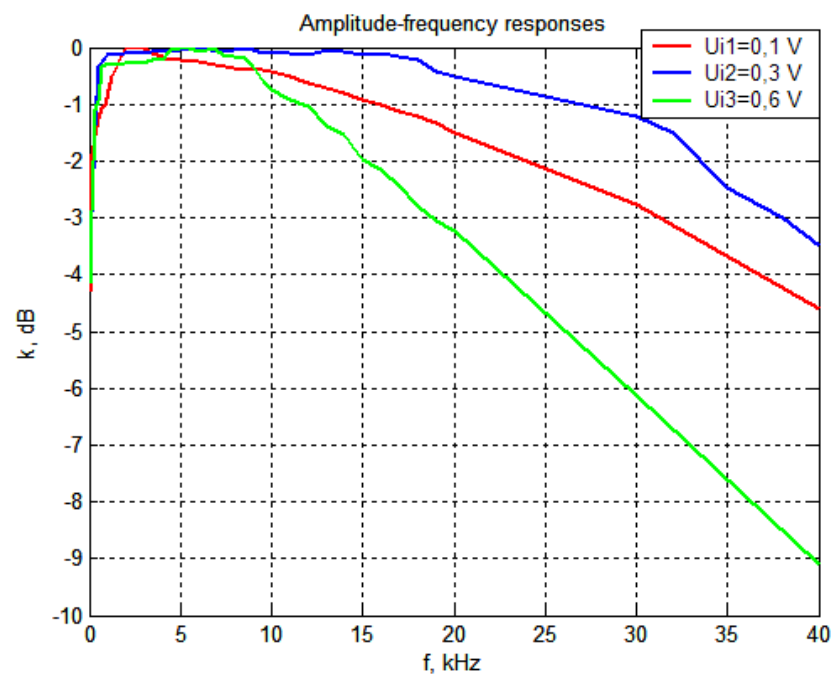

Figure 13. AFR of the LFPA Class D.

The maximum input voltage $u_{i m a x}$, which LFPA class D can amplify without distortion, is the same for lower and medium frequencies, and for higher ones its value slightly decreases. The sensitivity threshold of the circuit is the same for the operating frequencies studied and is in the order of 35 $\mathrm{mV}$.
Table 3. Qualitative Parameters of the LFPA Class D, Determined by AP.

\begin{tabular}{llll}
\hline Parameter & $\boldsymbol{f}_{\mathbf{1}}=\mathbf{0 , 1} \mathbf{~ k H z}$ & $\boldsymbol{f}_{\mathbf{2}}=\mathbf{0 , 5} \mathbf{~ k H z}$ & $\boldsymbol{f}_{\mathbf{3}}=\mathbf{1} \mathbf{~ k H z}$ \\
\hline $\mathrm{A}_{\mathrm{U}}$ & 26,3 & 27,1 & 26,8 \\
$\mathrm{u}_{\text {imax }}, \mathrm{V}$ & 0,68 & 0,68 & 0,5 \\
$\mathrm{u}_{\text {imin }}, \mathrm{V}$ & 0,035 & 0,035 & 0,035 \\
$\mathrm{D}$ & 19,4 & 19,4 & 14,2 \\
\hline
\end{tabular}

From Figure 13 it has been established that LFPA class D has steep slopes of AFR at low frequency. The gain factor for medium frequencies in the bandwidth is characterized by pulsations. The widest bandwidth is realized at the amplitude of the input signal $\mathrm{u}_{\mathrm{i} 2}=0,3 \mathrm{~V}$. In this case, the bandwidth is the widest as $\Delta f \approx 38 \mathrm{kHz}$.

From the parameters presented in Table 4 of the AFR of the LFPA class D it is established that the lower cut-off frequency $f_{\mathrm{b}}$ has the same value of $20 \mathrm{~Hz}$ and the value of $f_{\mathrm{h}}$ changes depending on the amplitude of the input signal as it receives the highest value at the average amplitude of the input signal. For $\mathrm{u}_{\mathrm{i} 3}=0,6 \mathrm{~V}$ the transmitted bandwidth is the narrowest and it is in the order of $19 \mathrm{kHz}$. It is established from the values obtained for $\Delta f$ (Table 4) that the implemented LFPA class D is Hi-Fi.

Table 4. Qualitative Parameters of the LFPA Class D, Determined by AFR.

\begin{tabular}{llll}
\hline Parameter & $\mathbf{u}_{\mathbf{i} 1}=\mathbf{0 , 1} \mathbf{~ V}$ & $\mathbf{u}_{\mathbf{i} 2}=\mathbf{0 , 3} \mathbf{~ V}$ & $\mathbf{u}_{\mathrm{i} 3}=\mathbf{0 , 6} \mathbf{~ V}$ \\
$f_{\mathrm{b}}, \mathrm{Hz}$ & 20 & 20 & 20 \\
$f_{\mathrm{h}}, \mathrm{kHz}$ & 32 & 38 & 19 \\
$\Delta f, \mathrm{kHz}$ & $\approx 32$ & $\approx 38$ & $\approx 19$ \\
\hline
\end{tabular}

In Figures from 14 to 23 are shown the timing diagrams obtained by means of a digital oscilloscope in the mentioned control nodes of the LFPA class D and the shape of the signals before and after the low-pass filter is shown in the time diagrams in Figure 24 (node 13) and Figure 25 (node 15) respectively.

Oscillograms are captured at the frequency of the input signal $f=1 \mathrm{kHz}$.

In Figure 14 and Figure 15 are shown the timing diagrams of the input harmonic signal supplied by the functional generator MCP SG2110 DDS used. Because OU1 is a repeater the signals have the same amplitude.

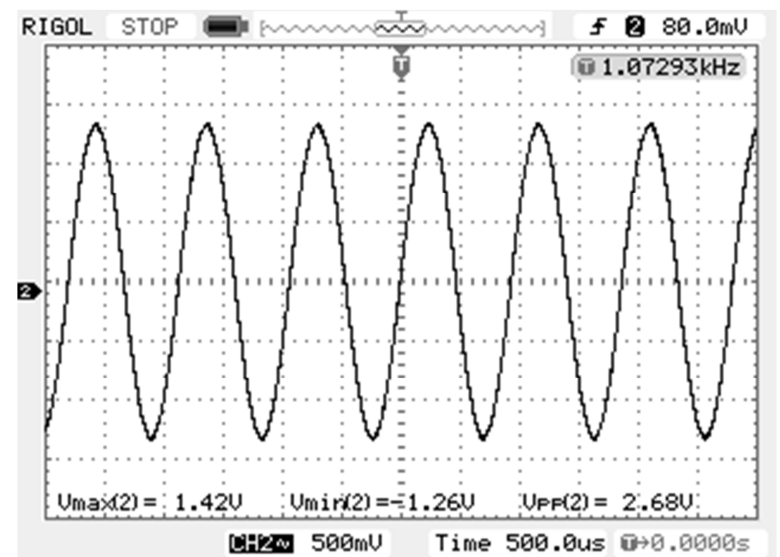

Figure 14. Timing Diagram of the Signal from the Setting Generator-Node 1 . 


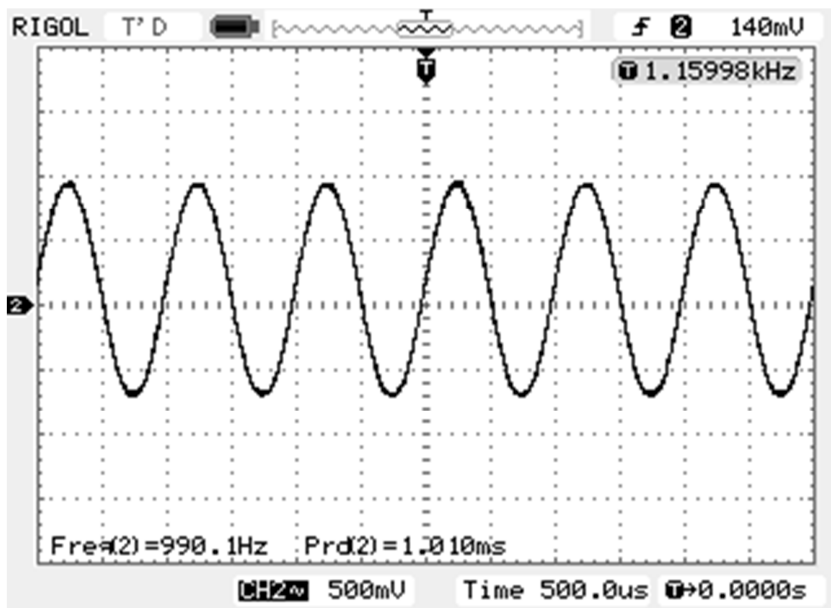

Figure 15. Timing Diagram of the Signal after OU1 - Node 2.

The type of signal in node 4 is shown in Figure 16 - OU3 performs the function of an adder-integrator as the two signals are sent to its non-inverting input: the continuous input and the high frequency PWM from the output via the inverse feedback circuit, implemented with the $\mathrm{R} 7$ resistor.

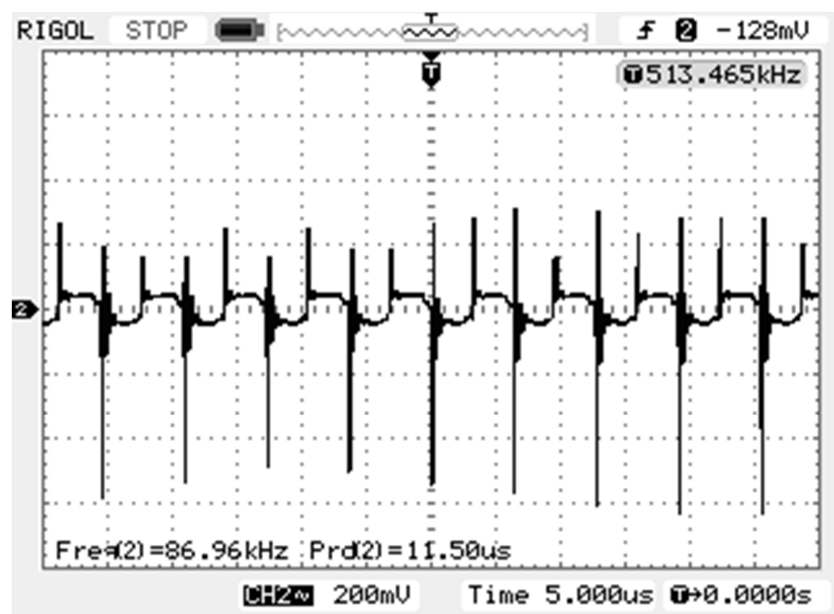

Figure 16. Timing Diagram of the Signal in Node 4.

The timing diagram of the signal after the adder-integrator (OU3, node 5) is shown in Figure 17. It has a triangular shape and has an operating frequency greater than $100 \mathrm{kHz}$. Rectangular pulses are formed at the output of the comparator - OU4 and their type is shown on the oscillogram in Figure 18. As a result of the presence of the R11 resistor and inverse feedback implemented with R12, Figure 19 shows the type of signal of the transistor bases Q1 and Q2 where the impulse mark/space ratio is greater than $50 \%$.

From the timing diagrams in nodes 9 and 10 (Figures 20 and 21), it was found that the control and also the output signals for the two pairs of complementary transistors have a different mark/space ratio - greater than and less than $50 \%$, respectively.

From the timing diagrams of Figure 22 and Figure 23 the difference in the control signals for the final MOSFET transistors is detected. They show that transistor Q7 has been unstopped during most of the PWM signal period at the output of the LFPA class D compared to Q8.

The output signal of LFPA class D, which is also an output of low-pass filter, is presented on the oscillogram in Figure 25. It has a sinusoidal shape and the presence of high frequency components is also established.

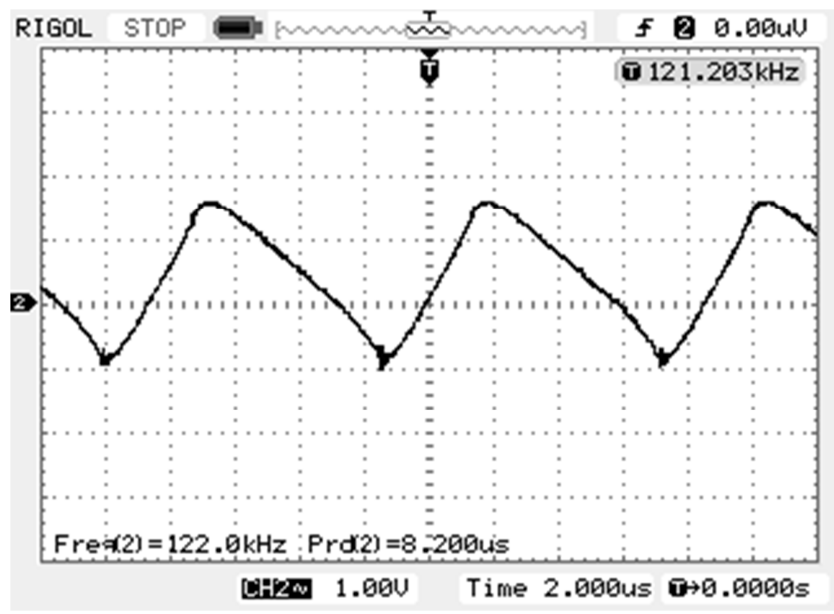

Figure 17. Timing Diagram of the OU3 Output - Node 5.

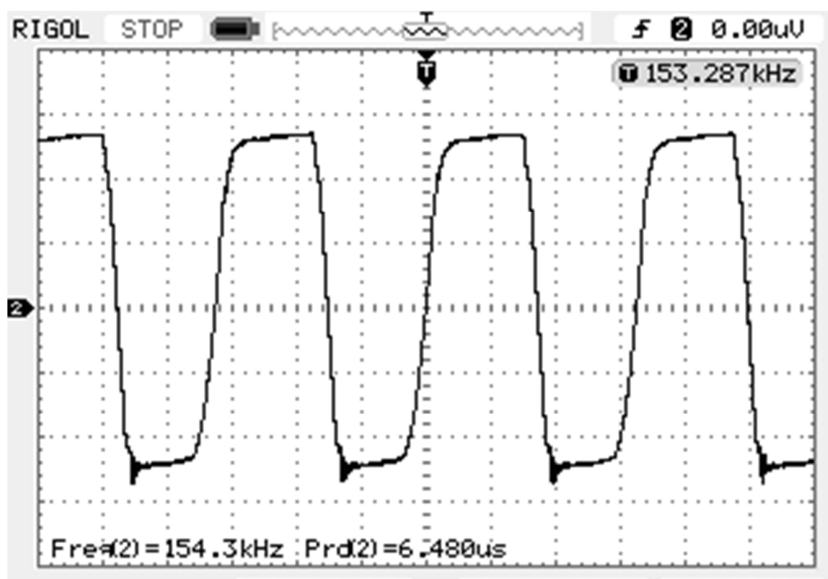

[CH2N 2.09U Time 2.000us Tab.0000s

Figure 18. Timing Diagram of the Signal after Comparator OU4 - Node 7.

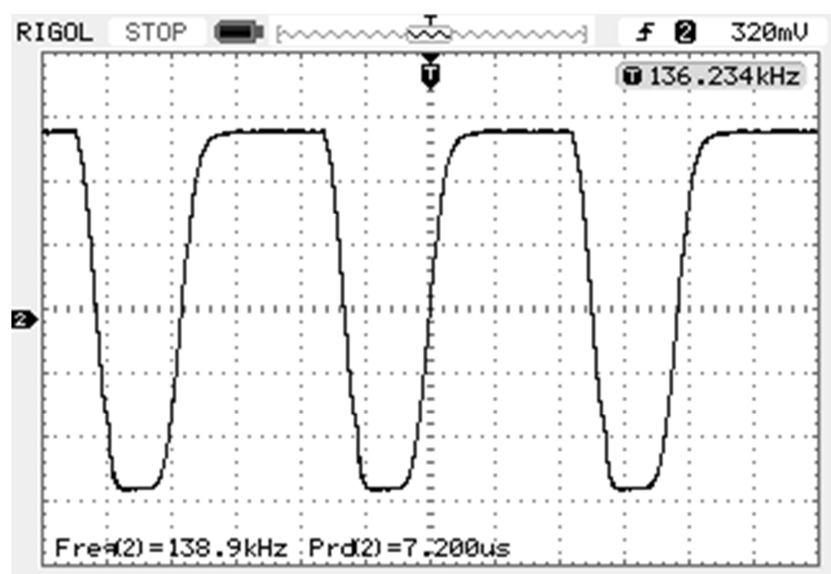

[CH2w 2.00U Time 2.000us Tra.0000s

Figure 19. Timing Diagram of the Bases of $Q_{1}$ and $Q_{2}$ - Node 8. 


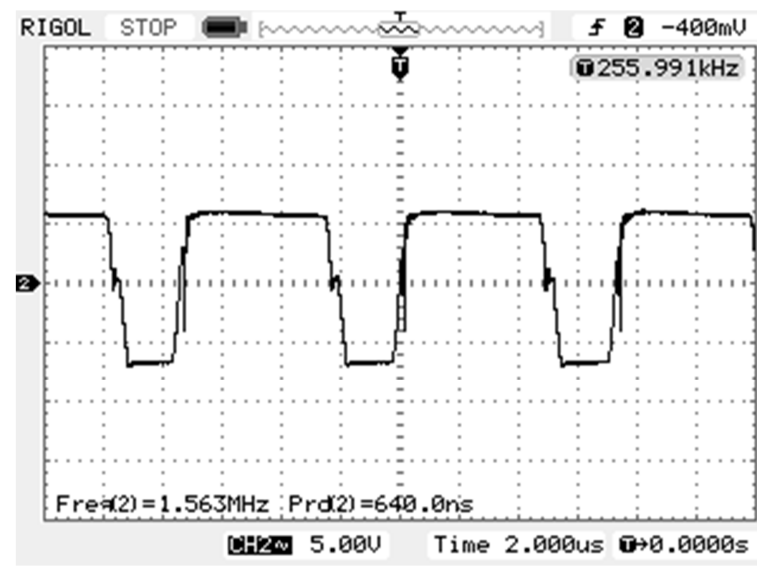

Figure 20. Timing Diagram of the Bases of $Q_{3}$ and $Q_{4}$ - Node 9.

In Figures $26 \div 29$ are shown the timing diagrams of the output PWM signal in node 13 of the LFPA class D at different amplitude of the continuous input signal and different input frequency. Using the digital oscilloscope RIGOL-DS1102E, the impulse parameters were measured, the values of which are presented in Table 5. It is found that at different amplitude of the input signal, the values of the parameters of the PWM signals (the pulses in node 13) are different, as is also different the "high" operating frequency. For the second value of the input voltage $\mathrm{U}_{\mathrm{i} 2}=0,3 \mathrm{~V}$ at $f=1$ $\mathrm{kHz}$ the impulse parameters:

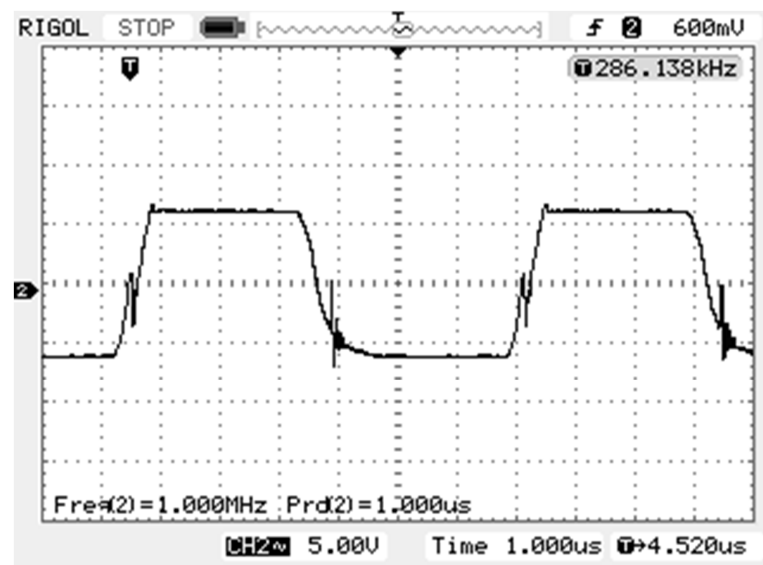

Figure 21. Timing Diagram of the Bases of $Q_{5}$ and $Q_{6}-$ Node 10.

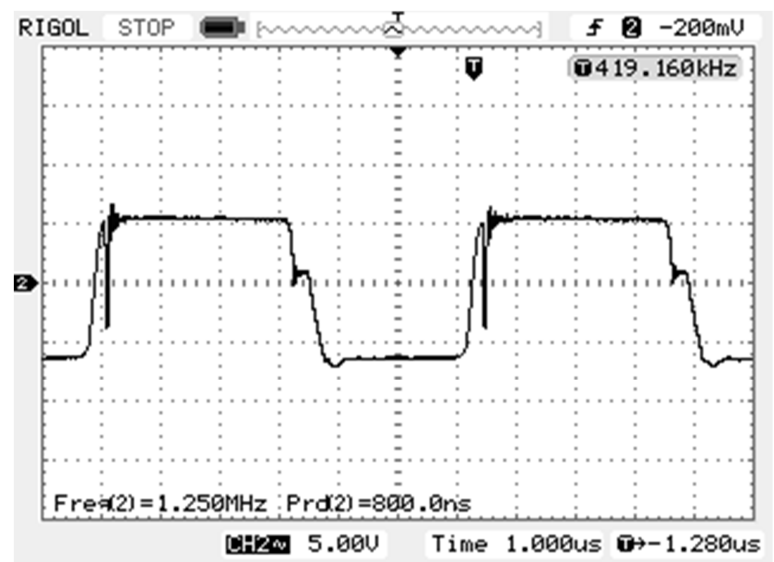

Figure 22. Timing Diagram of the Gate Signal of $Q 7$ - Node 11.

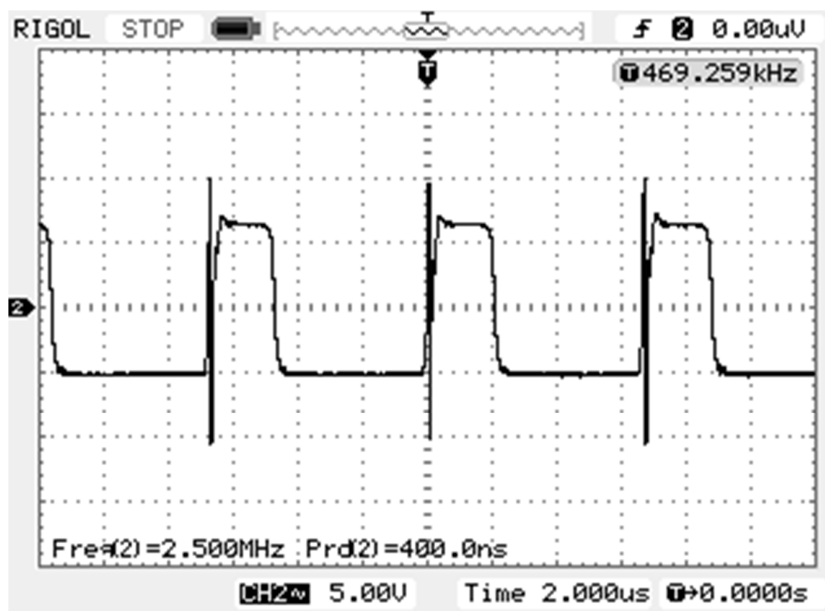

Figure 23. Timing Diagram of the Gate Signal of Q8 - Node 12.

$\mathrm{T}$ - period of impulse;

$\mathrm{T}_{\text {on }}$ - time of pulse rise;

$\mathrm{T}_{\text {off }}$ - time of pulse decrease;

$\mathrm{T}_{\mathrm{r}}$ - duration (time) of the inversion rebound of the impulse; $\delta_{\mathrm{f}}-$ rebound of the back front of the impulse

take the highest values.

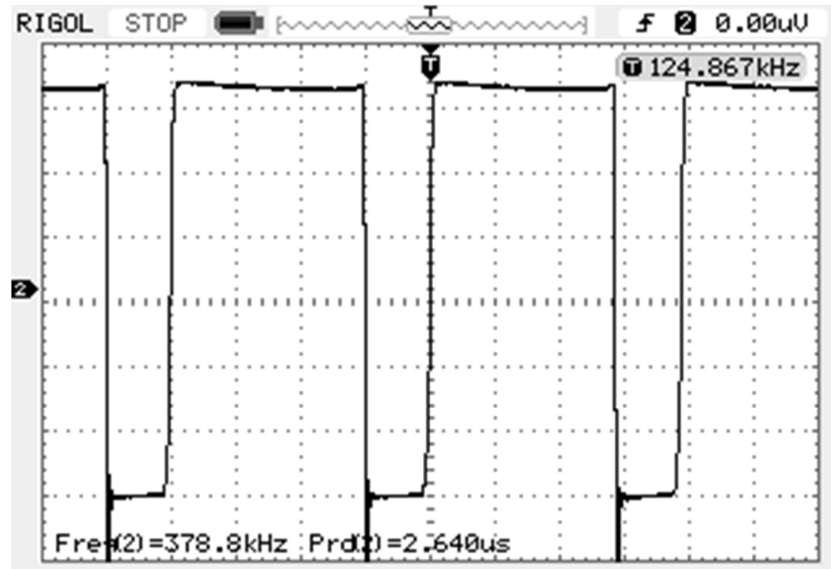

CH2N $10.0 \mathrm{~N}$ Time $2.000 \mathrm{~s}$ THด.0000s

Figure 24. Timing Diagram of the Signal Before Low-pass Filter - Node 13.

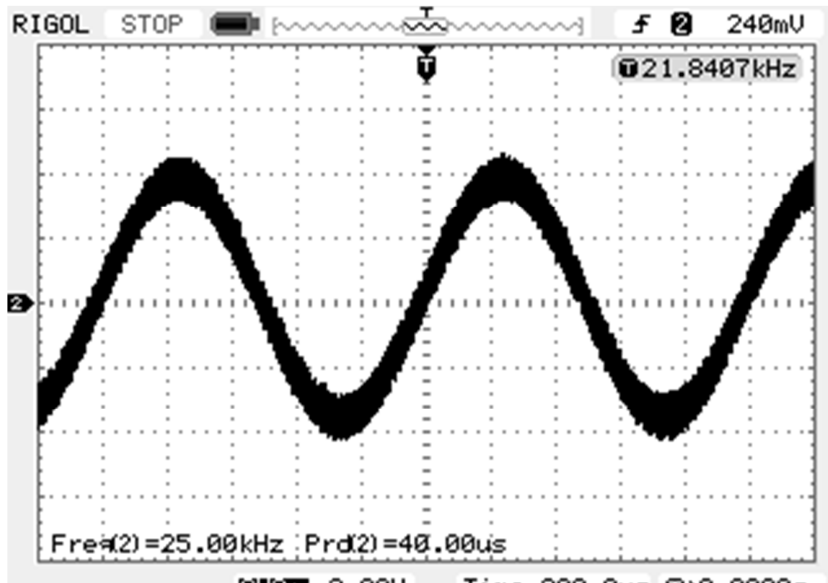

[CH2N 2.00U Time 200.0us Ta0.0000s

Figure 25. Timing Diagram of the Output of Low-pass Filter and LFPA Class D - Node 15. 


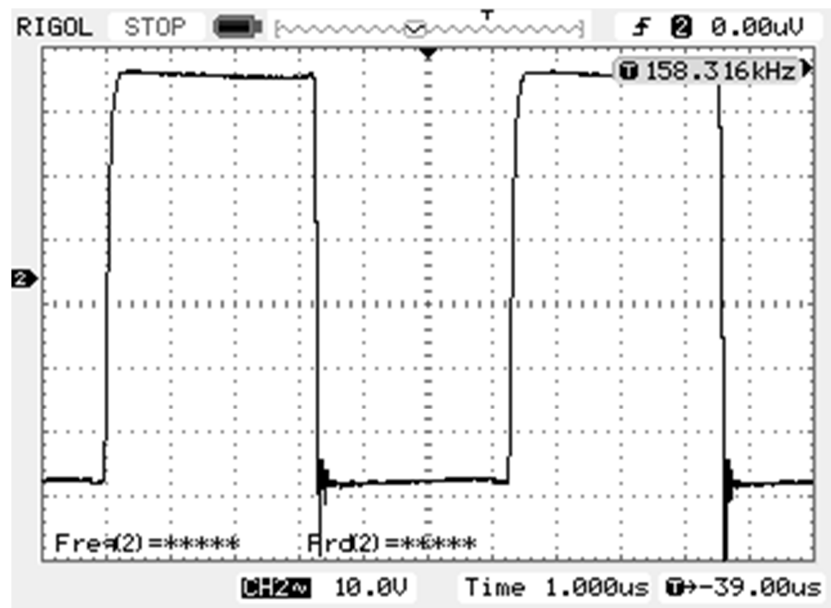

Figure 26. Timing Diagram of the Drains of $Q_{7}$ and $Q_{8}-$ Node 13 at $U_{i l}=$ $0,1 \mathrm{~V}$ and $f=1 \mathrm{kHz}$.

At a lower frequency of the input signal $f_{2}=100 \mathrm{~Hz}$ at the same voltage, the operating frequency of the PWM signals decreases and the values of the individual pulse parameters $\left(\mathrm{T}, \mathrm{T}_{\mathrm{on}}, \mathrm{T}_{\text {off }}, \mathrm{T}_{\mathrm{r}}\right.$ and $\left.\delta_{\mathrm{f}}\right)$ increase.

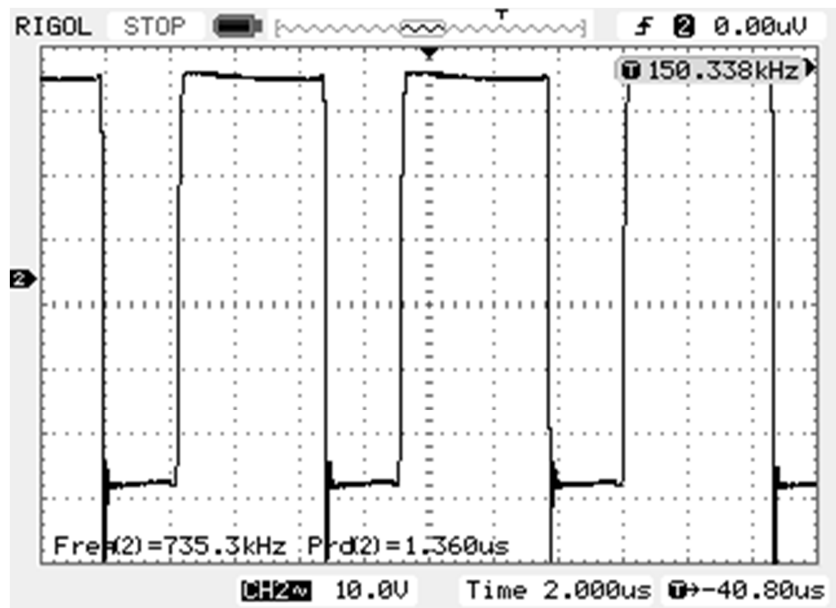

Figure 27. Timing Diagram of the Drains of $Q_{7}$ and $Q_{8}-$ Node 13 at $U_{i 2}=$ $0,3 \mathrm{~V}$ and $f=1 \mathrm{kHz}$

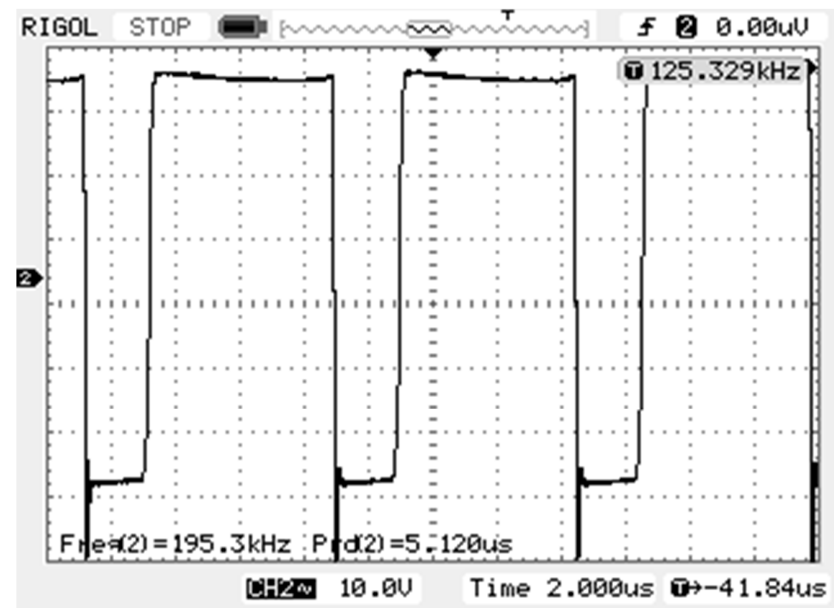

Figure 28. Timing Diagram of the Drains of $Q_{7}$ and $Q_{8}-$ Node 13 at $U_{i 3}=$ $0,6 \mathrm{~V}$ and $f=1 \mathrm{kHz}$.

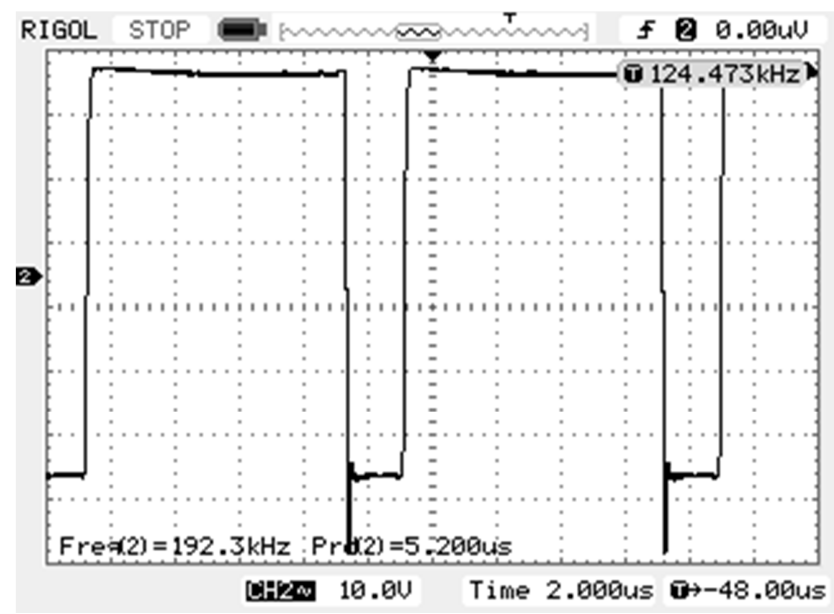

Figure 29. Timing Diagram of the Drains of $Q_{7}$ and $Q_{8}-$ Node 13 at $U_{i 3}=$ $0,6 \mathrm{~V}$ and $f=100 \mathrm{~Hz}$.

Table 5. Measured Parameters of the Output PWM Signal of the Drains of the Final MOSFET Transistors - Node 13.

\begin{tabular}{llllllll}
\hline & & $\mathrm{U}_{\mathrm{i} 1}$ & $\mathrm{U}_{\mathrm{i} 2}$ & $\mathrm{U}_{\mathrm{i} 3}$ & & & $\mathrm{U}_{\mathrm{i} 1}$ \\
& & $0,1 \mathrm{~V}$ & $0,3 \mathrm{~V}$ & $0,6 \mathrm{~V}$ & & & $0,6 \mathrm{~V}$ \\
$f_{1}=1$ & $\mathrm{~T}$ & $3,32 \mu \mathrm{s}$ & $5,24 \mu \mathrm{s}$ & $2,4 \mu \mathrm{s}$ & $f_{2}=$ & $\mathrm{T}$ & $3,18 \mu \mathrm{s}$ \\
$\mathrm{kHz}$ & $\mathrm{T}_{\text {on }}$ & $160 \mathrm{~ns}$ & $400 \mathrm{~ns}$ & $272 \mathrm{~ns}$ & 100 & $\mathrm{~T}_{\text {on }}$ & $320 \mathrm{~ns}$ \\
& $\mathrm{~T}_{\text {off }}$ & $320 \mathrm{~ns}$ & $336 \mathrm{~ns}$ & $264 \mathrm{~ns}$ & $\mathrm{~Hz}$ & $\mathrm{~T}_{\text {off }}$ & $304 \mathrm{~ns}$ \\
& $\mathrm{~T}_{\mathrm{r}}$ & $40 \mathrm{~ns}$ & $232 \mathrm{~ns}$ & $128 \mathrm{~ns}$ & & $\mathrm{~T}_{\mathrm{r}}$ & $208 \mathrm{~ns}$ \\
& $\delta_{\mathrm{f}}$ & $12,8 \%$ & $12,4 \%$ & $9,6 \%$ & & $\delta_{\mathrm{f}}$ & $12,8 \%$ \\
\hline
\end{tabular}

\section{Conclusion}

The presented schematics and the obtained simulation results explain the principle of operation and illustrate the operation of the LFPA class D shown in Figure 1, implemented with widespread and widely applied in practice discrete elements with low cost - operational amplifiers and transistors. They could be used to optimize its qualitative indicators - parameters and characteristics, and also provide expected practical results.

The experimental studies of the implemented LFPA class D with operational amplifiers and transistors present its qualitative parameters and characteristics and illustrate the type of signals in the individual nodes of the circuit as well. From them, information can be obtained about the causes of the accompanying LFPA class D deficiencies and to be done improvement of the technology used and they should be reduced to a minimum.

\section{References}

[1] B. Karapenev, "Fundamental Principles for Building Class D Audio- Amplifiers", Proceedings of the Union of Scientists Ruse, series of Technical Sciences, Volume: 11, ISSN 1311106X, 2014, pp. 36-41.

[2] B. Karapenev, A. Krumov, "Experimental studies of a implemented class D low power amplifier", Collection of papers, Volume: II, International Scientific Conference UNITECH'2015, Gabrovo, ISSN 1313-230X, 2015, pp. II-57II-62. 
[3] Chih-Min Chang, Jieh-Tsorng Wu, "A computationallyefficient PWM technique for digital class-D amplifiers", IEEE International Symposium on Circuits and Systems (ISCAS), 2016, pp. 1946-1949.

[4] D. McDonald, "Class D Audio-power Amplifiers: Interactive simulations assess device and filter performance", Transim Technology Corp., January 2001.

[5] G. Pillonnet, N. Abouchi, M. Chiollaz, P. Marguery, "Highperformance simulator for digital audio class D amplifiers", IET Circuits, Devices \& Systems, Volume: 4, Issue: 1, 2010, pp. $42-47$.

[6] J. Honda and J. Adams, "Class D Audio Amplifier Basics", Application Note AN-1071, International Rectifier, 2005.
[7] J. Honda, "Design Features - Energy Efficiency with Class D Amplifier Modules", Audio System Engineering International Rectifier, 2013.

[8] Tong Ge, J. Chang, "Modelling and Technique to Improve PSRR and PS-IMD in Analog PWM Class-D Amplifiers, IEEE Transactions on Circuits and Systems II: Express Briefs, Volume: 55, Issue: 6, 2008, pp. 512-516.

[9] Varona et al., "A Low-Voltage Fully-Monolithic $\Delta \Sigma$-Based Class-D Audio Amplifier", Proceedings of the 1999 European Solid State Circuits Conference, pp. 545-548.

[10] X. Jiang, "Fundamentals of Audio Class D Amplifier Design: A Review of Schemes and Architectures", IEEE Solid-State Circuits Magazine, Volume: 9, Issue: 3, 2017, pp. 14-25. 